\title{
LOS SISTEMAS DE INFORMACIÓN Y LA GENERACIÓN DE CONOCIMIENTO PARA LA GESTIÓN DE MARKETING
}

\section{Diana Forero (D.U.)}

\section{RESUMEN:}

Los sistemas de información de marketing (SIM) son de vital importancia para el desarrollo de las actividades y los procesos de decisión propios de la gestión del marketing dentro de las empresas. En las últimas décadas se ha ampliado su adquisición, pero presenta inconvenientes que han hecho cuestionar su aplicabilidad y conformidad con las necesidades reales de los gestores. Parte de estas inconformidades se deben a una inadecuada conceptualización del nivel de decisión que está implicado en los SIM, debido, en muchos casos, a que son ubicados como sistemas funcionales u operativos más que como sistemas estratégicos, lo que conlleva al empleo de tecnologías inadecuadas para el tipo de información que se requiere y los propósitos buscados por los usuarios. Además, los SIM tienen una función en la generación de conocimiento de mercadeo que implica prácticas de gestión y de trabajo colaborativo que no son las tradicionales en el desarrollo de software de gestión transaccionales por lo que se generan dificultades en la interacción de los grupos. En el artículo se proponen elementos de análisis de la problemática y algunas sugerencias para mejorar estas interacciones y, por lo tanto, los resultados de los SIM para las empresas.

\section{ABSTRACT:}

Marketing information systems (MIS) are really important for the development of activities and for the decision pro- cesses that belong to the marketing management in the companies. In the last decades the acquisition of these systems has increased. This has brought problems that have questioned their applicability and conformity with the real needs of agents. Part of this nonconformity is due to an inadequate conceptualization of the decision level implied in the MIS. This is because the MIS are placed as functional or operative systems instead of as strategic systems. For this reason, inadequate technologies are used for the type of information required as well as for the purpose the user wants. In addition, the function of the MIS in the generation of marketing knowledge implies management and collaborative work practice, which are not traditional when developing transactional management software, creating problems in the group interaction. In the article, we suggest elements for the analysis of the problem and some ways to improve not only these interactions, but also the results of the MIS for the companies.

\section{PALABRAS CLAVE:}

Sistemas de información de marketing, gestión del conocimiento, desarrollo de software, inteligencia de mercados.

\section{KEYWORDS:}

Marketing Systems of Information, Knowledge Administration, Software Development, Markets Intelligence.

\section{INTRODUCCIÓN}

Las empresas han optado por la sistematización de muchos de sus procesos de marketing con el fin de generar información para una mejor gestión. Los sistemas de información de marketing (SIM) han ido ganando espacios en los diferentes procesos de gestión, pero en la medida en que crece su uso tam- bién generan cuestionamientos en los directivos. Es común encontrarse con gerentes de marketing que se preguntan ¿cómo sacarle más provecho a sus bases de datos?, ¿deben invertir en otro sistema o no?, ¿cómo hacer para que los empleados usen un sistema que fue adquirido?, ¿cómo lograr que los ingenieros entiendan las necesidades reales de 
Un sistema de información puede definirse como un conjunto de componentes interrelacionados que permiten capturar, procesar, almacenary distribuir la información para apoyar la toma de decisiones yel control en una institución mercadeo frente al SIM?, estas entre otras preguntas revelan que el problema de la sistematización de la información de marketing no es solo el de poseer SIM's.

En este escrito se busca mostrar que las empresas se están enfrentando simultáneamente a los problemas de sistematización y desarrollo de SIM, generación de información y generación de conocimiento para la gestión de marketing por lo cual se requiere revisar otros aspectos adicionales para lograr un mejor resultado en su administración.

\section{SIM Y GESTIÓN DE MARKETING}

Un sistema de información puede definirse como un conjunto de componentes interrelacionados que permiten capturar, procesar, almacenar y distribuir la información para apoyar la toma de decisiones y el control en una institución ${ }^{1}$. Para que los sistemas de información generen una solución adecuada a las necesidades de la gerencia, se sigue un proceso de solución de problemas en donde se identifica la brecha entre la situación actual y la deseada, se evalúan los recursos disponibles y el desarrollo requerido para dar la solución ${ }^{2}$. Este proceso de diseño se realiza teniendo en

\section{RESEÑA AUTOR:}

Diana Forero (D.U.) Psicóloga de la Universidad Nacional de Colombia, especialista en análisis de datos de la Universidad de la Salle y Master en Ciencias de Gestión de la Universidad de Rouen, Francia. Estudiante del programa de Doctorado en Ciencias de la Gestión, de la misma universidad. Es autora de varios artículos en los campos de la psicología y el mercadeo en revistas como Suma Psicológica, Panorama y P\&M. Actualmente desarrolla su trabajo investigativo en temas que relacionan el comportamiento del consumidor y la estrategia de mercadeo. Correo electrónico dforero@poligran.edu.co. cuenta que existen diferentes niveles de decisión y que los sistemas de información deben responder a las necesidades que se imponen en cada nivel.

Los teóricos en el campo de los sistemas de información generalmente reconocen tres niveles jerárquicos en los procesos de decisión de las organizaciones: el de las decisiones estratégicas (dirección general), el de las decisiones tácticas (dirección funcional) y el de las decisiones operativas (dirección operativa). Para cada uno de ellos se trabajan distintos sistemas de información ${ }^{3}$.

De acuerdo con esta clasificación de los sistemas de información, los SIM corresponderían principalmente al nivel funcional. Para los sistemas de este nivel se asigna la responsabilidad de proveer información necesaria para la planificación táctica, el control y la toma de decisiones de corto plazo; es decir, aquella información que incide de manera inmediata sobre la rentabilidad de la empresa ${ }^{4}$. Algunas otras actividades de la gestión de marketing se encontrarían en el nivel operativo, como las bases de datos de clientes, la información de transacciones y de despachos, entre otras.

Esta clasificación de los sistemas de información tiene mucha utilidad práctica, pero no corresponde a la forma como se asume la gestión del marketing actualmente, en donde se tiene un marketing estratégico y uno operativo ${ }^{5}$ y aparece un marketing holístico donde está involucrada la totalidad de la organización, los clientes, los socios comerciales y los stakeholders ${ }^{6}$. La gestión de marketing, en consecuencia, involucra información de los

1. Laudon, K. y Laudon J. (1996). Administración de los sistemas de información. México: Prentice Hall, pág. 8.

2. O’Brien, J. (2004). Sistemas de información gerencial. Bogotá: McGraw-Hill, pág, 81.

3. Gil, I. (1997). Sistemas y tecnologías de la información para la gestión. Madrid: McGraw Hill.

4. Ibíd

5. Lambin, J. (1985). Marketing estratégico. México: McGraw Hill, cap.1

6. Kotler, P. (2004). El marketing se mueve. Buenos Aires: Paidós, cap. 2. 
tres niveles planteados por los teóricos de los sistemas de información gerencial, aunque actualmente se les ubique en los dos niveles inferiores.

De otra parte, como se verá en el siguiente cuadro, la información requerida para la gestión de marketing implica carac- terísticas diferenciales de los SIM debido a las fuentes de información y a la información misma. Algunos de estos requisitos son comunes a todos los sistemas de información, sin embargo se señalan aquellos que son considerados por la autora como de mayor importancia:

Cuadro 1. Gestión del marketing y características requeridas de los SIM

\begin{tabular}{|c|c|c|c|}
\hline $\begin{array}{l}\text { Gestión de } \\
\text { marketing }\end{array}$ & $\begin{array}{l}\text { Características de } \\
\text { la información }\end{array}$ & Fuentes de información & $\begin{array}{l}\text { Características requeridas de } \\
\text { los sistemas de información }\end{array}$ \\
\hline $\begin{array}{l}\text { Marketing } \\
\text { estratégico }\end{array}$ & $\begin{array}{l}\text { No programada } \\
\text { Ocasional } \\
\text { Resumida } \\
\text { De amplio alcance } \\
\text { Difícil acceso }\end{array}$ & $\begin{array}{l}\text { Internas } \\
\text { Proveedores de } \\
\text { información } \\
\text { Asociaciones, entidades } \\
\text { públicas } \\
\text { Medios } \\
\text { Informales }\end{array}$ & $\begin{array}{l}\text { A la medida } \\
\text { Flexibles } \\
\text { Adaptables } \\
\text { Acceso limitado } \\
\text { Alta capacidad para seleccionar } \\
\text { información }\end{array}$ \\
\hline $\begin{array}{l}\text { Marketing } \\
\text { operativo }\end{array}$ & $\begin{array}{l}\text { Especificada } \\
\text { previamente } \\
\text { Abundante } \\
\text { Frecuente } \\
\text { Histórica } \\
\text { Geográfica }\end{array}$ & $\begin{array}{l}\text { Internas } \\
\text { Proveedores de } \\
\text { información } \\
\text { Clientes } \\
\text { Canales } \\
\text { Fuerza de ventas } \\
\text { Web, etc. }\end{array}$ & $\begin{array}{l}\text { Alta capacidad de almacenamiento } \\
\text { y procesamiento de datos } \\
\text { Automatizados } \\
\text { Fácil acceso y administración } \\
\text { Funcionamiento en red } \\
\text { Conectividad } \\
\text { Fácil desarrollo de aplicaciones } \\
\text { Integrabilidad con otros sistemas }\end{array}$ \\
\hline $\begin{array}{l}\text { Marketing } \\
\text { holístico }\end{array}$ & $\begin{array}{l}\text { Relacional } \\
\text { Cambiante } \\
\text { Histórica } \\
\text { Personalizada } \\
\text { Focalizada } \\
\text { Refiere a procesos o } \\
\text { a personas }\end{array}$ & $\begin{array}{l}\text { Clientes } \\
\text { Proveedores } \\
\text { Socios comerciales } \\
\text { Stakeholders } \\
\text { Otras áreas, } \\
\text { departamentos o divisiones } \\
\text { de la empresa }\end{array}$ & $\begin{array}{l}\text { Funcionamiento en red } \\
\text { Facilitar el trabajo colaborativo } \\
\text { A la medida } \\
\text { Alta capacidad de almacenamiento } \\
\text { y generación de informes } \\
\text { Conectividad } \\
\text { Permitir el seguimiento a } \\
\text { informaciones puntuales } \\
\text { Facilitar la personalización de la } \\
\text { información }\end{array}$ \\
\hline
\end{tabular}

Desde la perspectiva del marketing estratégico y del marketing holístico la información tiene carácter estratégico y por tal motivo los sistemas de información no sólo deben tener un sentido de visualizar las acciones de la competencia, el sector y el entorno, sino desarrollar toda una inteligencia del mercado y del negocio. Los sistemas de información que dan respuesta a estas necesidades tienen altos requerimientos técnicos para su desarrollo, y por lo tanto, son costosos y tardan bastante tiempo en ser desarrollados, como es el caso de los sistemas 
Los sistemas

de informa-

ción para el

marketing ope-

rativo se han

desarrollado

ampliamente y

se encuentran

desde sistemas

de administra-

ción de bases

de datos hasta

sistemas de

automatización

de oficinas, sis-

temas de pro-

cesamiento de

información de

escáneres, sis-

temas de inteli-

gencia artificial

$y$ sistemas de

información

por redes como

internet. expertos y los sistemas de información para ejecutivos (EIS), por tal motivo, tienen un mayor riesgo de obsolescencia una vez se han desarrollado y requieren un mayor trabajo colaborativo para que no pierdan su enfoque. En estos sistemas se integran elementos estratégicos y tácticos de las compañías al construirse la "inteligencia del mercado", con información interna y externa y con fuentes poco estructuradas.

De otra parte, el marketing holístico requiere sistemas de información colaborativos y en red, y desarrolla desde las tecnologías de información como intranets, extranets, groupware, sistemas de comunicación electrónica, sistemas integrados y los sistemas de intercambio electrónico de datos (EDI), entre otros. Estos sistemas de información implican redes dentro y fuera de la empresa y son poco comunes en los desarrollos actuales de los SIM. Por el tipo de información que proveen son de carácter estratégico, no solo para la empresa, sino para los distintos componentes y actores de las redes de negocios.

Los sistemas de información para el marketing operativo se han desarrollado ampliamente y se encuentran desde sistemas de administración de bases de datos hasta sistemas de automatización de oficinas, sistemas de procesamiento de información de escáneres, sistemas de inteligencia artificial y sistemas de información por redes como internet. El nivel de sofisticación de estos sistemas es muy variado y en general, son los primeros en adoptarse en las empresas. Estos sistemas son abundantes en el mercado, pero no resuelven los grandes interrogantes de la gestión de mar- keting; requieren análisis de datos dispendiosos y tienden a dar una mirada introspectiva de la empresa con pocos elementos de la realidad que la circunda.

Desde este punto de vista, aunque existe una incongruencia sobre los alcances de los SIM en la teoría respecto a la práctica de la gestión de marketing, se observa que también existe una respuesta positiva de los desarrolladores de software frente estas necesidades. Sin embargo, aunque el esfuerzo de los desarrolladores es meritorio continúan los serios cuestionamientos. Allí cabe hacerse la pregunta ¿por qué?. Si existen las tecnologías y existen los desarrollos ¿por qué no se han logrado alinear los SIM con la gestión?

El problema no es solamente que existan las tecnologías de información y la posibilidad de desarrollo de los SIM en sus diferentes niveles de complejidad, también radica en que en muchos casos no es claro cuál es el aporte real de cada uno de estos sistemas a la gestión de marketing, en parte porque no se sabe en qué sistemas invertir sin tener un exceso de información o de gasto o llegar a la inoperatividad; en otros, porque no se sabe cómo usar lo disponible y sacarle el máximo provecho. El aporte de los SIM no se puede evidenciar solamente desde los reportes que estos permiten o desde la promesa del desarrollador de hacer aplicaciones cada vez más específicas, es necesario tener en cuenta otros aspectos de la generación del conocimiento que están implícitos en su adopción.

\section{SIM Y GESTIÓN DEL CONOCIMIENTO}

Los sistemas de información no solo proveen datos, permiten la generación de 
información y de conocimiento. Autores como Barabba y Zaltman ${ }^{7}$ o Rowley ${ }^{8}$ sugieren que la función de todo sistema de información en marketing debe ser la de generar conocimiento que permita acercarse a la mejor decisión, a la mejor estrategia. Para que se desarrolle conocimiento no es suficiente con poseer el sistema, éste debe transformar los datos en información y la información debe transformarse en conocimiento. En ambos casos los SIM son insuficientes pues se requiere la intervención humana. Aunque se pueden simular las decisiones, finalmente quien decide cuál es un dato sin importancia y cuándo hay una información a considerar es una persona y a su vez, la decisión de qué información genera un conocimiento la toma uno o varios gestores. Generar un conocimiento va más allá de poseer la información.

El problema de la generación de conocimiento es que puede tener la participación de uno o varios individuos, grupos o hasta organizaciones y la transformación de la información dada por el sistema implica procesos perceptuales subjetivos y conocimientos tácitos, de allí la complejidad de lograr que se dé el proceso completo sin que parezca que el SIM arroja demasiado o muy poco.

Siendo éste un problema complejo, las soluciones también lo son, algunos autores consideran que parte de lo que deben aprender las organizaciones es a aceptar que la fluctuación y el cambio son parte de la gestión del conocimiento ${ }^{9}$, por lo tanto, se esperaría que los gestores y los sistemas de información manejen esa incertidumbre, teniendo a la vez flexibilidad y precisión. Sin duda el reto tecnológico es crear sistemas que aprendan por sí mismos y que puedan encontrar caminos para el conocimiento y los procesos de decisión desde información poco estructurada y variable, es decir, contextual.

La organización también debe tener mayor cuidado en usar el conocimiento de su propio negocio en la definición de sus requerimientos de información para optimizar el desarrollo de los SIM y los requerimientos tecnológicos. En este sentido debe definirse claramente la intención del sistema y permitir la participación de los usuarios en su definición y desarrollo. Esto implica un llamado no sólo a lo interdisciplinar, sino a la cualificación de los usuarios que tienen incidencia directa en la definición de los sistemas.

De otra parte, existe una clara necesidad de manejar procesos de capacitación y de gestión del cambio tanto con los desarrolladores como con los gestores de marketing para establecer lenguajes comunes, generar trabajo colaborativo y sobre todo, para convertir conocimientos tácitos en explícitos. Una buena parte del avance de los sistemas dependerá del desarrollo de capacidades conjuntas y de crear modelos de trabajo colaborativo que permitan detectar aspectos claves que manejan de manera individual los responsables de marketing y con ello mejorar el modelamiento y el desarrollo de
La organización también debe tener mayor cuidado en usar el conocimiento de su propio negocio en la definición de sus requerimientos de información para optimizar el desarrollo de los SIM y los requerimientos tecnológicos.

7. Varaba, V. y Zaltman, G. (1992). La voz del mercado. México: McGraw Hill \& Harvard Business School

8. Rowler, J. (2002). Reflections on customer knowledge management in e-business. Bradford: Qualitative Market Research.Tomo5, No 4; pág. 268

9. Nonaka, I. y Takeuchi, H. (1999). La organización creadora de conocimiento. Oxford University Press. 
los sistemas. Esto no es posible si dentro de las empresas se perciben unos y otros como dos grupos aparte, con distintas responsabilidades y donde los resultados del sistema no tienen dolientes comunes que puedan retroalimentar el proceso y que hayan participado desde su definición.

Como conclusión se sugiere que las empresas, al examinar los problemas a los que se enfrenta cuando implementa SIM consideren estos últimos aspectos: la gestión del conocimiento, la definición con el usuario de las aplicaciones concretas de los SIM frente a los procesos de gestión del marketing y los conocimientos del propio negocio y la gestión del cambio y el trabajo colaborativo.

\section{BIBLIOGRÁFIA}

- Gil, I. (1997). Sistemas y tecnologías de la información para la gestión. Madrid: McGraw Hill.

- Kotler, P. (2004). El marketing se mueve. Buenos Aires: Paidós, cap. 2.

- Lambin, J. (1985). Marketing estratégico. México: McGraw Hill, cap.1

- Laudon, K. y Laudon J. (1996). Administración de los sistemas de información. México: Prentice Hall, pág. 8.

- Nonaka, I. y Takeuchi, H. (1999). La organización creadora de conocimiento. Oxford University Press.

- O`Brien, J. (2004). Sistemas de información gerencial. Bogotá: McGraw-Hill. pág, 81.

- Rowler, J. (2002). Reflections on customer knowledge management in e-business. Qualitative Market Research. Bradford.Tomo5, No 4; pg. 268

- Varaba, V. y Zaltman, G. (1992). La voz del mercado. México: McGraw Hill y Harvard Business School. 